\title{
STORAGE RINGS: PAST, PRESENT AND FUTURE
}

\author{
A.D. Krisch* \\ Randall Laboratory of Physics, University of Michigan \\ Ann Arbor, Michigan 48109-1120
}

This lecture will attempt to review storage rings: past, present and future. I will spend more time on the past, because the past has produced most of our data, while the present can be rather brief. There is not yet much future data, but there are some plans about what we hope for. Professor Andy Sessler of Berkeley, who recently reviewed the early history of colliders [1], loaned me his slides; for this I have much appreciation.

Storage rings can either collide a stored particle beam with a second stored particle beam or they can store particles for collisions with internal targets. The recent Accelerator Handbook, edited by A. W. Chao and M. Tigner [2], contains a list of all colliders above $1 \mathrm{GeV}$; this list, which is shown in Fig. 1, mostly contains electron and proton colliders. Since Professor Muenzenberg just told us about some important advantages in having electron on heavy ion colliders, perhaps the next edition of this handbook will include more nuclear facilities.

\begin{tabular}{|c|c|c|c|}
\hline Location & Name (type) & Max. $E_{c m}(\mathrm{GeV})$ & Start \\
\hline \multirow[t]{5}{*}{ Stanford/SLAC,USA } & $C B X^{[b]}\left(e^{-} e^{-} D R\right)$ & 1.0 & 1963 \\
\hline & Spear $\left(e^{+} e^{-} \mathrm{SR}\right)$ & 5.0 & 1972 \\
\hline & $\mathrm{PEP}\left(e^{+} e^{-} \mathrm{SR}\right)$ & 30 & 1980 \\
\hline & $\operatorname{SLC}\left(e^{+} e^{-} \operatorname{LC}\right)$ & 100 & 1989 \\
\hline & PEP-II $\left(e^{+} e^{-}\right.$DR $)$ & 10.6 & 1999 \\
\hline \multirow[t]{3}{*}{ Frascati, Itali } & $\operatorname{AdA}\left(e^{+} e^{-} \mathrm{SR}\right)$ & 0.5 & 1962 \\
\hline & Adone $\left(e^{+} e^{-} \mathrm{SR}\right)$ & 3.0 & 1969 \\
\hline & $\mathrm{DA} \Phi \mathrm{NE}\left(e^{+} e^{-} \mathrm{SR}\right)$ & 1.0 & 1997 \\
\hline \multirow[t]{3}{*}{ Novosibirsk, Russia } & VEP-1 $\left(e^{+} e^{-} \mathrm{DR}\right)$ & 0.26 & 1963 \\
\hline & VEPP-2/2M $\left(e^{+} e^{-}\right.$SR $)$ & 1.4 & 1974 \\
\hline & VEPP-4 $\left(e^{+} e^{-} \mathrm{SR}\right)$ & 14 & 1979 \\
\hline Cambridge, USA & CEA Bypass $\left(e^{+} e^{-}\right.$SR $)$ & 6 & 1971 \\
\hline \multirow{2}{*}{ Orsay, France } & $\mathrm{ACO}\left(e^{+} e^{-} \mathrm{SR}\right)$ & 1.0 & 1966 \\
\hline & $\mathrm{DCI}\left(e^{ \pm} e^{ \pm} \mathrm{DR}\right)$ & 3.6 & 1976 \\
\hline \multirow[t]{3}{*}{ DESY, Germany } & Doris $\left(e^{+} e^{-} \mathrm{DR}\right)$ & 6.0 & 1974 \\
\hline & Petra $\left(e^{+} e^{-} \mathrm{SR}\right)$ & 38 & 1978 \\
\hline & $\mathrm{HERA}\left(e^{ \pm} p\right.$ DR $)$ & 160 & 1992 \\
\hline \multirow[t]{4}{*}{ CERN, Europe } & ISR (pp DR) & 63 & 1971 \\
\hline & SppS (p̄ SR) & 630 & 1981 \\
\hline & $\operatorname{LEP}\left(e^{+} e^{-\mathrm{SR}}\right)$ & 190 & 1989 \\
\hline & LHC (pp DR) & 14000 & 2004 \\
\hline Brookhaven, USA & $\begin{array}{l}\text { RHIC (heavy ions DR) } \\
\text { RHIC (pp DR) }\end{array}$ & $\begin{array}{c}200 / u \\
500\end{array}$ & 1999 \\
\hline & $\operatorname{CESR}\left(e^{+} e^{-\mathrm{SR}}\right)$ & 12 & 1979 \\
\hline \multirow{2}{*}{ KEK, Japan } & Tristan $\left(e^{+} e^{-} \mathrm{SR}\right)$ & 60 & 1986 \\
\hline & KEK B $\left(e^{+} e^{-} \mathrm{DR}\right)$ & 10.6 & 1999 \\
\hline Beijing, China & $\mathrm{BEPC}\left(e^{+} e^{-} \mathrm{SR}\right)$ & 3.1 & 1989 \\
\hline Fermilab, USA & Tevatron (pp $\mathrm{SR})$ & 1000 & 1987 \\
\hline
\end{tabular}

Fig. 1 List of Colliders [2]

* Supported by a Research Grant from the U.S. Department of Energy

CP512, Nuclear Physics at Storage Rings, edited by H.-O. Meyer and P. Schwandt ㄷ 2000 American Institute of Physics 1-56396-928-9/00/\$17.00 
In his lectures on early colliders, Professor Sessler asked the question, "How did it happen?"; then he pointed out that for many years people knew that it would be much easier, because of relativity, to reach very high center-of-mass energies by having two moving particles collide head-on. He also noted that the famous Norwegian physicist, Wideroe, patented this idea during the War years; however, he apparently never received any money for his patent. Wideroe also apparently invented the LINAC, but that is another story which is not related to STORI99.

Sessler stressed that Donald Kerst, Gersh Budker and Bruno Touschek each played a very important role in storage rings; he loaned me some nice pictures of these three distinguished gentlemen. Donald Kerst is shown in Fig. 2; he was the senior author of the first paper proposing colliding beams and he also invented the betatron. He was a very distinguished scientist who died in 1993; somehow, he did not get a Nobel Prize; I do not understand why. Gersh Budker is shown in Fig. 3; he was the founder of the Novosibirsk Institute and he led the construction of a series of colliders at Novosibirsk, which have been quite important. Bruno Touschek is shown in Fig. 4 along with one of his junior colleagues. He is the only one of the three that I never met; he was very active in the pioneering AdA and Adone facilities in Frascati, Italy.

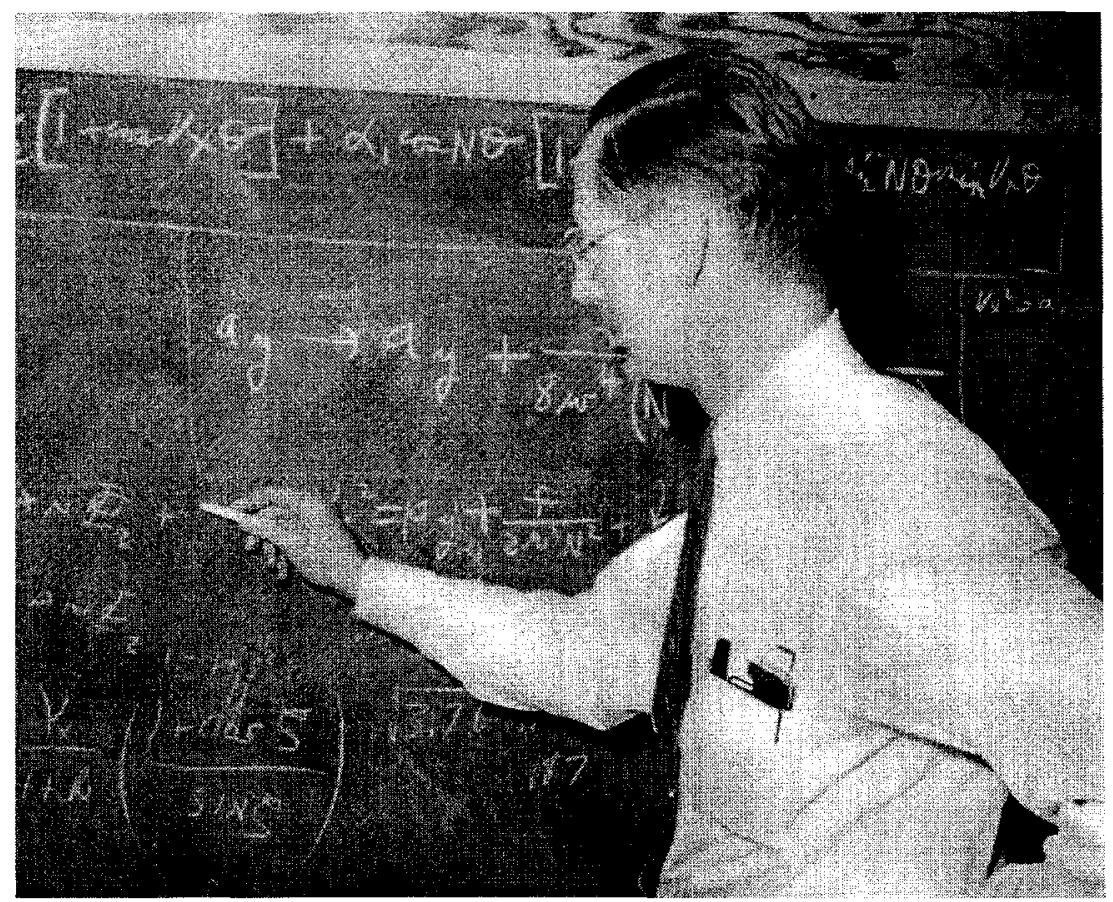

Fig. 2 Donald Kerst ${ }^{\dagger}$ 


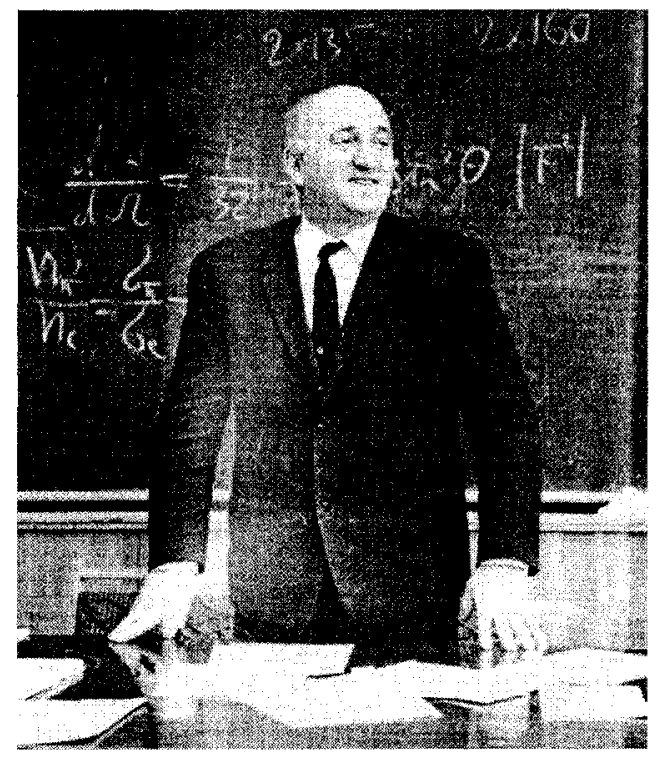

Fig. 3 Gersh Budker ${ }^{\dagger}$

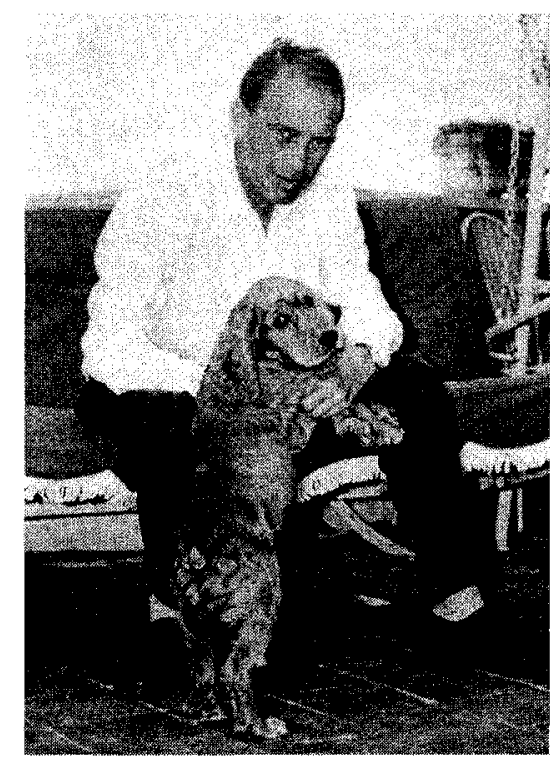

Fig. 4 Bruno Touschek ${ }^{\dagger}$

For colliders to work properly, the strong focusing principle needed to be invented. This is true because, for two beams to collide with a reasonably high collision rate, each beam must have a high brightness. Until about 1960, most accelerators were of the weak focusing type with beams that were rather large and defuse. The weak focusing accelerators worked fairly well for fixed target experiments, but it was clear that they would not work well for colliders because of their low brightness. Fortunately three very bright people, Courant, Livingston and Snyder, proposed in 1952 the strong focusing principle [3]; essentially all modern accelerator rings have been built using this strong focusing principle. Strong focusing is not strictly related to colliders, but colliders probably would not have worked without it. In 1956, some people at the Midwest Universities Research Association (MURA) proposed the somewhat related Fixed Field Alternating Gradient particle accelerators in another attempt to increase the brightness of large diffuse beams [4].

The development of colliding beams and storage rings started with a 1956 Physical Review paper by a group at MURA which was led by Kerst [5]. Sessler considers this a landmark paper along with another landmark paper [6] by Kjell Johnsen, a second Norwegian, who was the first Director of the world's first high energy proton collider, the ISR. The first page of the Kerst et al. paper is shown in Fig. 5; the other authors are: F. T. Cole, H. R. Crane, L. W. Jones, I. J. Laslett, T. Ohkawa, A. M. Sessler, K. R. Symon, K. M. Terwilliger and N. V. Nilsen. Three of these authors, Crane, Jones and Terwilliger, I know well because they were at Michigan; the others authors were mostly at other mid-western universities. This paper, which was published in April 1956, soon led to many other papers. 
Figure 6 shows the first page of an April 11, 1956 paper [7] that was written by G. K. O'Neill at Princeton, apparently just a few days after the Kerst et al. paper was published. O'Neill's idea was to produce colliding beams not by having two accelerators touching tangentially at one collison point, as Kerst et al. proposed, but instead by extracting two beams and then making the two beams collide. This technique was recently used at the SLAC Linear Collider (SLC). Moreover, this technique is now being considered for the huge proposed multi-billion dollar lepton colliders called the TESLA Linear Collider, the Next Linear Collider, the New Linear Collider or the Nippon Linear Collider.
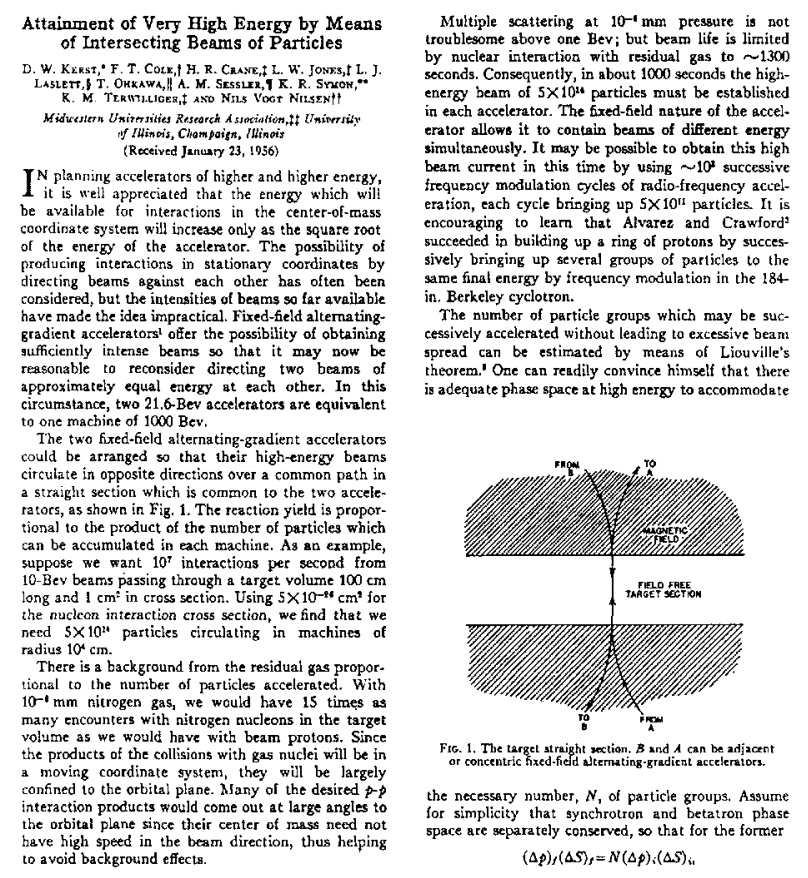

Fig. 5 First Colliding Beam Paper [5]

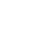

.

.

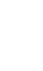

.

.


The first page of another important colliding beam paper [8], written by Donald Lichtenberg, Roger Newton and Marc Ross, is shown in Fig. 7; all three were then professors here at Indiana and also members of MURA. I am pleased to see Professor Lichtenberg in the audience. They proposed to use a single accelerator, but to somehow use its magnets to steer the particles so that they could collide. This technique is now used in many existing colliders; for example, Fermilab's Tevatron-Collider has only one ring but it contains two counter-rotating beams, one beam of protons and the other beam of antiprotons. For some reason these three gentlemen never published this paper; however, I had heard about it and Professor Lichtenberg recently gave me a copy of it and O'Neill's paper.

Professor Sessler gave me some nice photos of early accelerator and storage rings. Figure 8 shows the MURA Mark I FFAG, which was called the Michigan Model. This accelerator looks a bit like a cyclotron, but it did exhibit a type of strong focusing. The FFAG technique has not really been used very much, probably for the same reason that one would not want to build an SSC sized cyclotron: because then one must fill much of the area inside the ring with iron. The MURA Two Way Model, which was perhaps the first operating collider, is shown in Fig. 9.

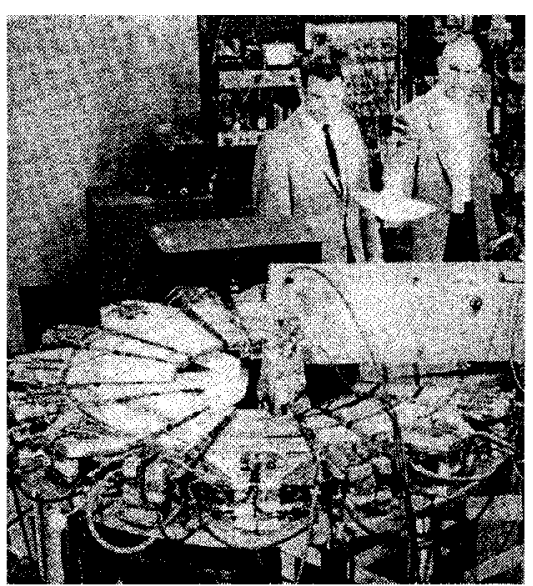

Fig. 8 MURA Mark I FFAG Michigan Model ${ }^{\dagger}$

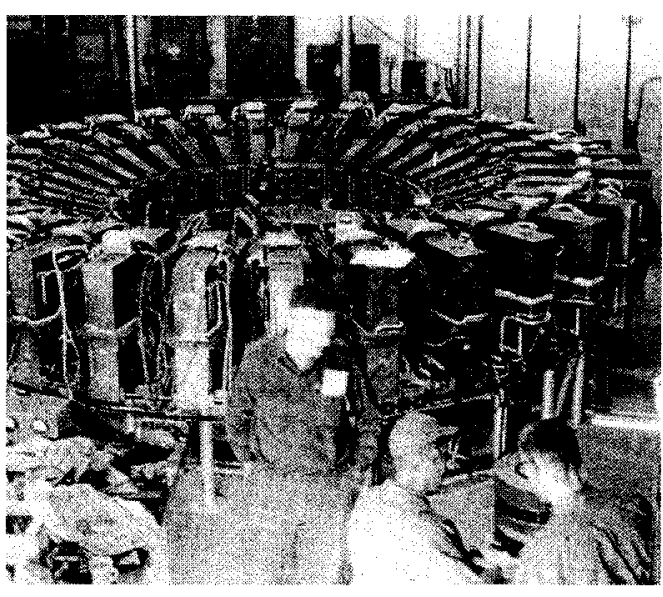

Fig. 9 MURA Two Way Model Collider ${ }^{\dagger}$

The first Stanford electron-electron collider, which started operating in 1959, is shown in Fig. 10; this was clearly using the idea originally proposed by Kerst et al. of having two physically separate accelerator rings with beams which collide where they touch. Figure 11 shows AdA, which Touschek built along with his colleagues at Frascati, in March 1961; AdA was the first electron-positron collider. 


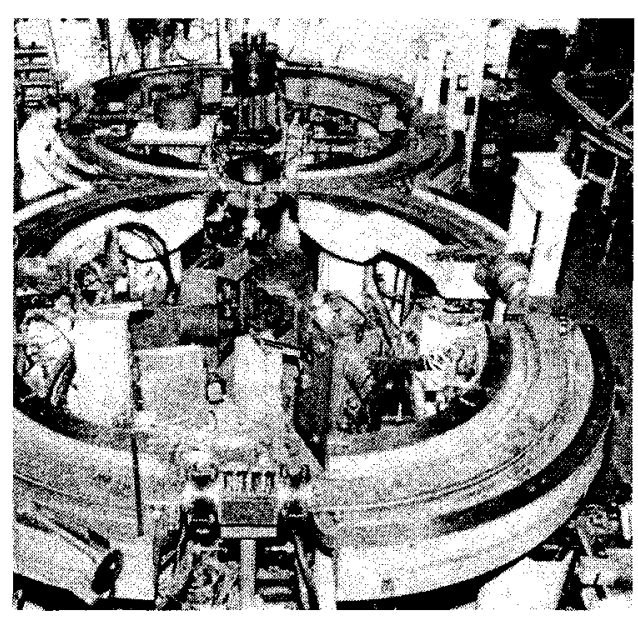

Fig. 10 First Stanford e-e Collider ${ }^{\dagger}$

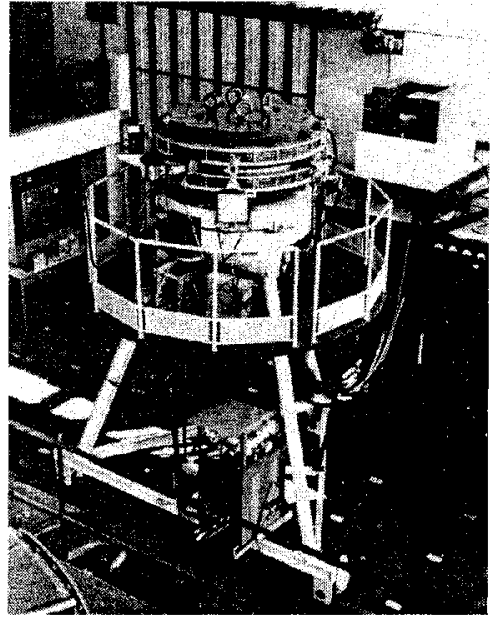

Fig. 11 AdA at Frascati (1961) $)^{\dagger}$

During the 1960s many people were enthusiastically building colliders. The first Novosibirsk electron-positron storage ring VEEP 2 is shown in Fig. 12 in 1967. Perhaps this electron-positron collider was the first use of the technique of Lichtenberg, Newton, and Ross, where one made two particles from a single ring somehow collide. VEEP 4 is the present collider at the Budker Institute of Nuclear Physics (BINP) in Novosibirsk; it has had an active program of experiments.

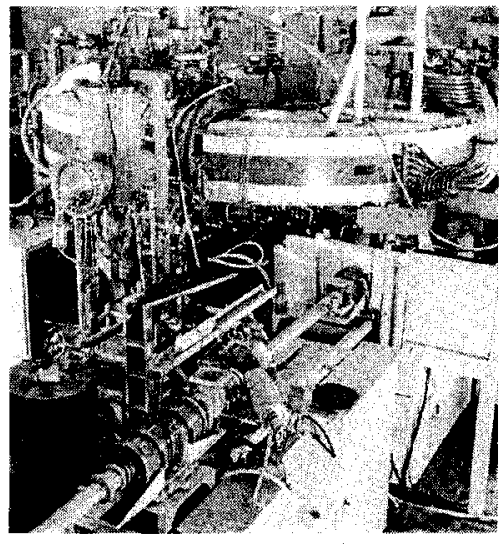

Fig. 12 VEEP 2 at Novosibirsk ${ }^{\dagger}$

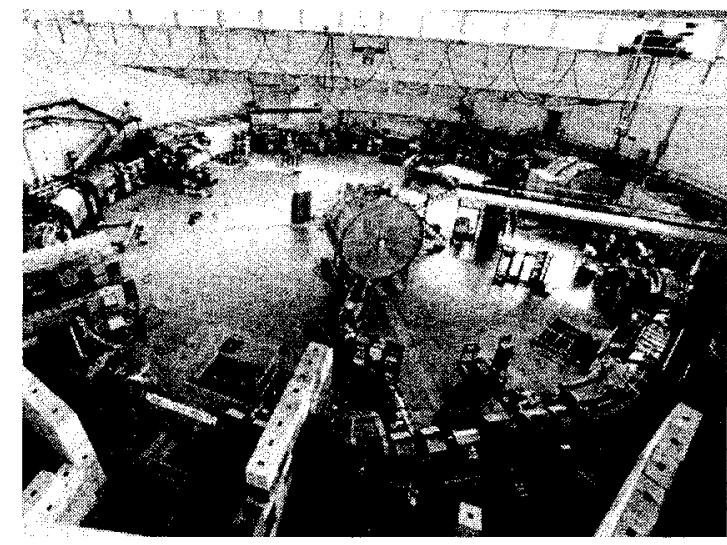

Fig. 13 ADONE at Frascati ${ }^{\dagger}$

ADONE, which was the first "large" electron-positron storage ring, is shown in Fig. 13; its energy was 1 or $2 \mathrm{GeV}$. There is a special problem with circular electron storage rings at very high energy, where their synchrotron radiation becomes very 
large; therefore, one must inject a great deal of RF acceleration energy to compensate for these synchrotron radiation energy losses. Moreover, one needs many RF stations, distributed uniformly around the ring, to inject the RF into the ring uniformly and thus avoid the large energy loss that can occur in a single turn.

The electron model for the ISR, around 1960, is shown in Fig. 14. Kjell Johnsen and his colleagues built this small-scale model of ISR with electrons rather than protons.

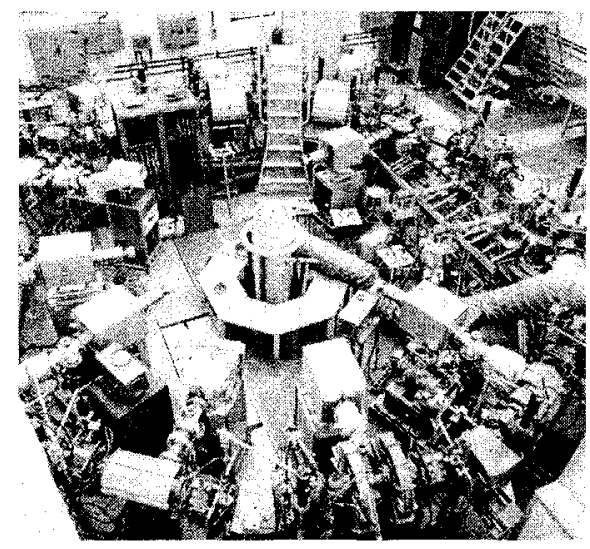

Fig. 14 Electron Model for ISR ${ }^{\dagger}$

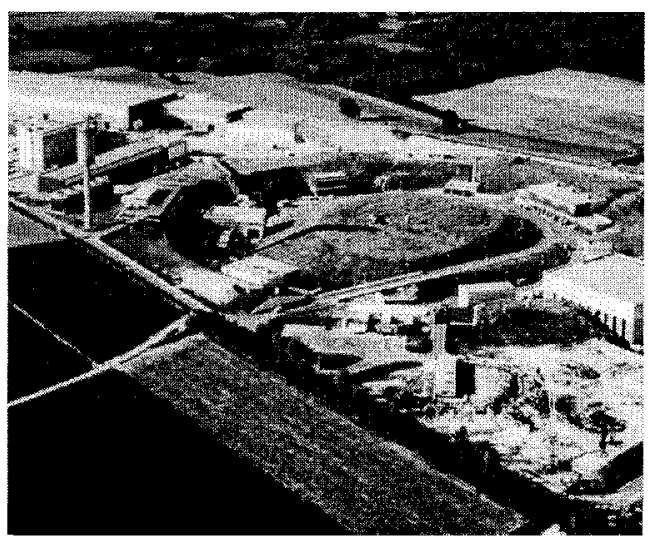

Fig. 15 The CERN ISR (1971)

Now I will diverge from Andy Sessler's talk, because I worked on the ISR when it first operated in 1971. Figure 15 shows a photo of ISR, which first extended CERN from Switzerland into France; the ISR's injector was the venerable PS which is also shown. I remember that we had to be very careful not to go into France by accident without showing our passports. Figure 16 shows a diagram of the ISR and the PS; the PS would alternately inject protons into each ISR ring. Notice the eight different crossing regions where the two ISR beams crossed in the horizontal plane.

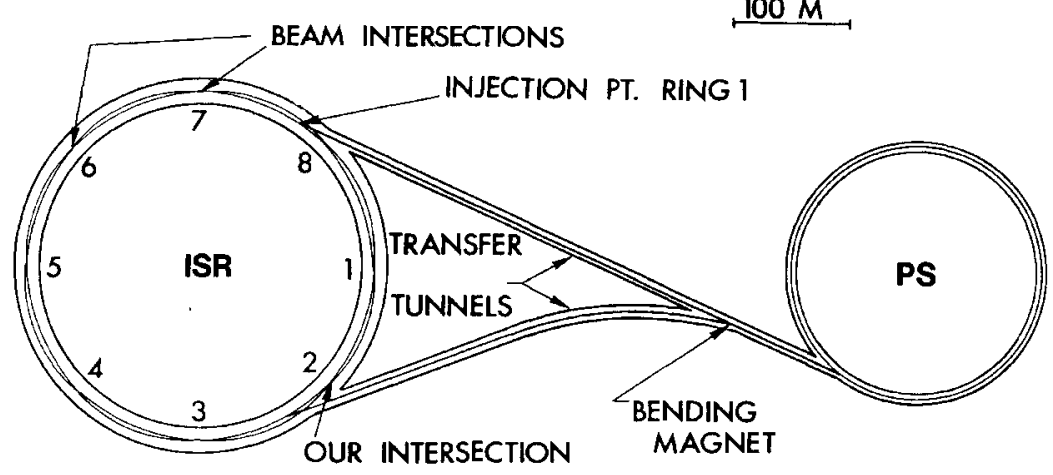

Fig. 16 CERN ISR and PS [6] 
Figure 17 shows the I 2 crossing region where we did the ABM experiment, which studied inclusive scattering using a narrow 43-meter-long spectrometer containing three bending magnets and a few scintillation and Cherenkov counters. We measured inclusive cross sections at $1500 \mathrm{GeV}$ laboratory energy equivalent; that was a huge energy jump from the pre-1971 maximum energy of $30 \mathrm{GeV}$. Moreover, many people expected to find real physical quarks at the ISR. Therefore, this was a very exciting time.

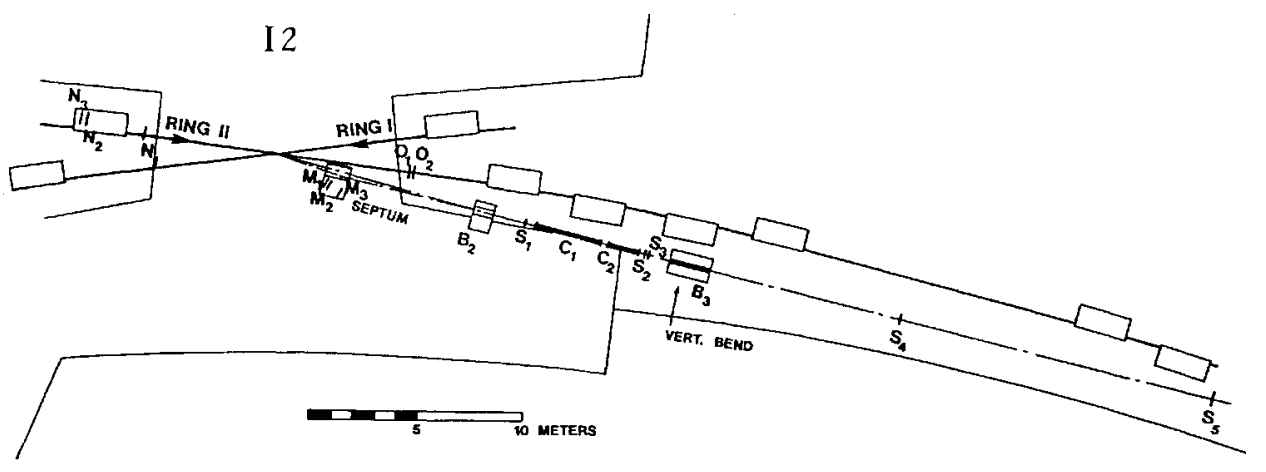

Fig. 17 ISR's I 2 Region with Argonne-Bologna-Michigan Inclusive Experiment

The ISR was an absolutely beautiful machine; it may be the best accelerator facility that will ever be built. It started operating six months ahead of time and apparently reached its design value before it was supposed to first operate. Eventually, the ISR reached a luminosity of about two orders of magnitude above its design value.

We did the first experiment at the ISR, which was slightly strange, because we were mostly an American group at the first really forefront European accelerator. Perhaps the reason was that I have always been an optimist; thus, all our equipment was ready about six months ahead of time and then suddenly the ISR started running. Most other ISR experimenters, such as Carlo Rubbia, were off doing something else; then suddenly the ISR beam was operating and they did not have any working detectors. The year 1971 was great fun and was clearly one of the high points of my career.

The I 2 crossing region is again shown in Fig. 18; notice the ISR ring magnets and the front end of our spectrometer which started with a special septum magnet that we built and brought to CERN. Michigan paid for this septum magnet which was assembled and tested at Argonne by Larry Ratner and Jim Bywater. We had to convince Kjell Johnsen and his colleagues that the septum's fringe field would not destroy the ISR beam, which was only a few centimeters from the septum's corner. 


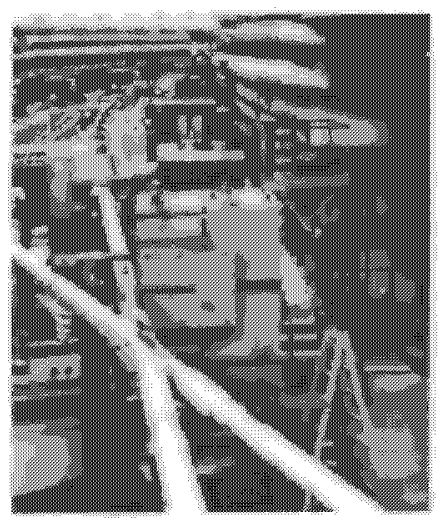

Fig. 18 ABM Septum Magnet

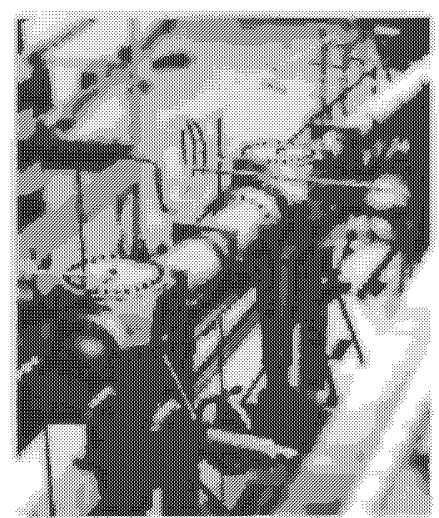

Fig. 19 ABM Cherenkov Counters

The Cherenkov counters in our spectrometer, which were provided by CERN, are shown in Fig. 19. We had our own Cherenkov counters at Michigan, but we used CERN Cherenkov counters because these detectors were under ten or twenty atmospheres of pressure and they could turn into rockets. My friends in Washington were concerned that, if an American-made Cherenkov counter destroyed the ISR, there could be a lot of trouble. Probably the CERN people were also concerned; in any case, they provided the Cherenkov Counters. We brought the other detectors from Michigan; these were standard scintillation counters mounted on transit stands with plumb bobs to make sure that they did not move.

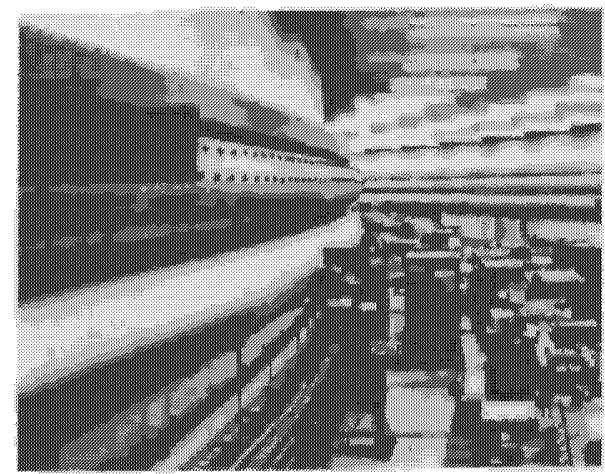

Fig. 20 End of ABM Spectrometer

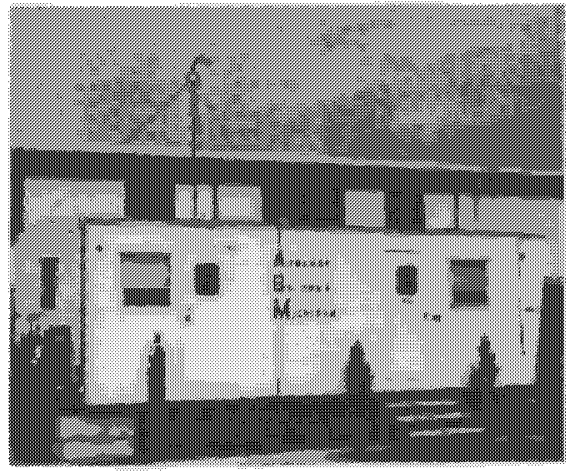

Fig. 21 ABM Trailer

The curvature of the ISR tunnel can be seen in Fig. 20, along with our spectrometer's final magnet, which was bending vertically up. Also notice our two downstream scintillation detectors on high extended transit stands. Fig. 21 shows our experimental counting house which was then called a trailer. Our ABM Collaboration involved Argonne, Bologna and Michigan; in 1971 there was a fierce debate in the world about anti-ballistic missiles, so we had fun with the name ABM. Our counting house's interior is shown in Fig. 22; notice the state-of-the-art electronics from 28 years ago and a pulse-height analyzer, which was already old in 1971. 


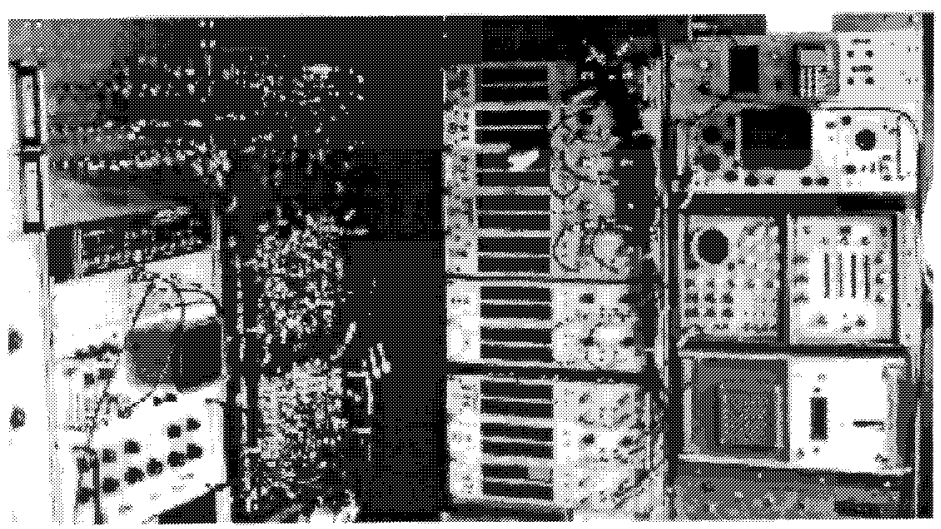

Fig. 22 Inside the ABM Counting House (Trailer)

When the first ISR program was approved, everyone was very concerned about how to measure the luminosity; no one had ever done an experiment with proton storage rings and there was no plan about how to measure luminosity. Our experiment planned to measure inclusive cross sections at very high energy to test the scaling law, which had been proposed independently by Feynman and by Yang. Therefore, we certainly needed an absolute measurement of the luminosity; otherwise, we could not calibrate the $1500 \mathrm{GeV}$ inclusive cross sections to compare them with the 12 and $24 \mathrm{GeV}$ data from the Argonne ZGS [9] and the CERN PS [10]. Every experimenter was quite concerned about this luminosity problem and this concern certainly reached the ISR staff.

To measure the luminosity, a very smart ISR staff member named Simon van der Meer invented the idea of using some small magnets to shift the beam vertically in small steps: the first magnet would bend the beam vertically up; the next magnet would bend it back into the horizontal plane; then, after the interaction region, the third magnet would bend the beam down and the fourth magnet would realign the beam along the original orbit. These four magnets were wired together in series; thus, when one increased the current by perhaps $1 \mathrm{amp}$, the beam would just move up perhaps 1 millimeter in the interaction region. Figure 23 shows the event rate in our luminosity monitors plotted against the vertical separation in millimeters. This variation of the separation allowed one to measure the effective height $h_{\text {eff }}$ of the two ISR beams where they collided. This $h_{\text {eff }}$ value allowed an absolute collaboration of the luminosity using the equation:

$$
\text { Luminosity }=\frac{I_{1} I_{2}}{h_{e f f} \cos \alpha}
$$

where $I_{1}$ and $I_{2}$ are the intensities of the two beams and $\alpha$ is their crossing angle in the horizontal plane. Thus, Dr. van der Meer allowed us all to measure absolute cross section. I do not recall meeting him in 1971; perhaps Kjell Johnsen and Franco Bonaudi told me of his idea. I recall thinking that van der Meer must be very clever; some years later he did something else clever and got the Nobel Prize. 


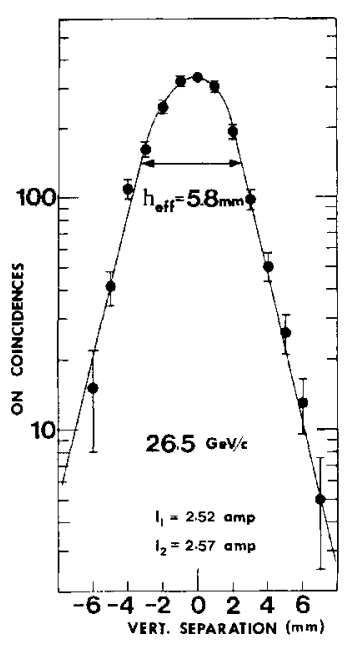

Fig. 23 van der Meer Curve with Small Beams

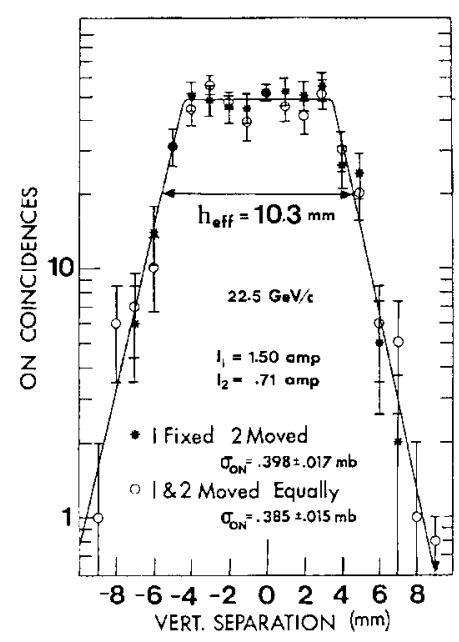

Fig. 24 van der Meer Curve with Large Beams

Note that in Fig. 23, the effective height of the beams was 5.8 millimeters, while it was 10.3 millimeters in Fig. 24, when the beams were much larger. Figure 24 also contains some tests that we did together with the ISR staff; in one case, one beam was fixed while the other beam was moved; in the second case the two beams were moved equally. Notice that the two sets of data agree perfectly; this helped to confirm that the van der Meer Technique really did work.

These first ISR inclusive results were published in Physical Review Letters [11] on 5 July 1971, the paper was submitted 2 June. In 1974 we published a detailed Physical Review paper [12]. Figure 25 shows our ISR inclusive cross section data at effective energies of 500 , 1100 and $1500 \mathrm{GeV}$ plotted against $P_{\perp}^{2}$ and $X_{F}$ together with the lower energy data. Notice that we measured the inclusive production of $\pi^{+}$ mesons, $K^{+}$mesons and protons, while our Bologna collaborators later published inclusive data on $\pi^{-}, K^{-}$

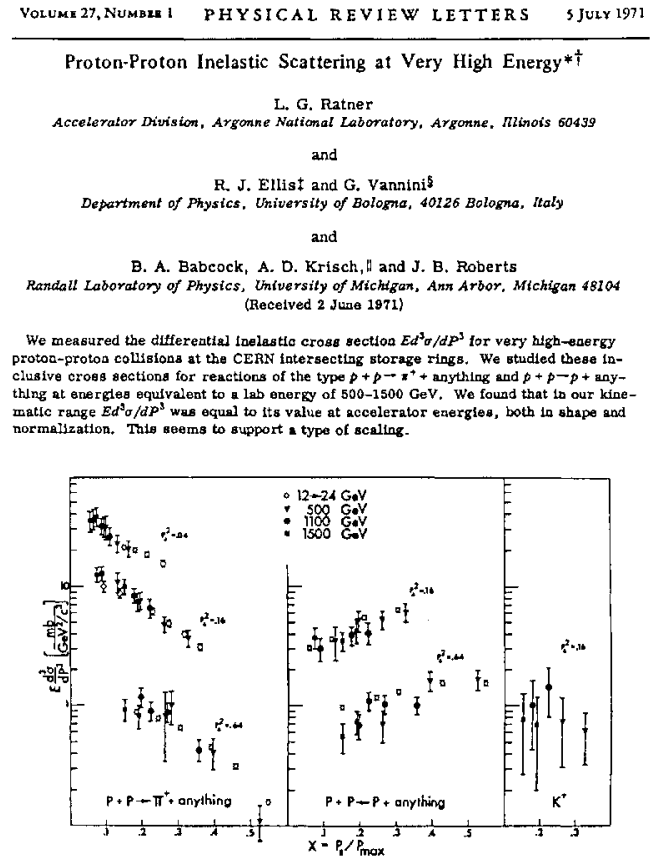

Fig. 25 First Evidence for FeynmanYang Inclusive Scaling [11,12] 
and antiproton production [13]. Our inclusive data points at 500, 1100 and 1500 $\mathrm{GeV}$ fall right on top of each other; moreover, they fall right on top of our earlier $12 \mathrm{GeV}$ ZGS and the $24 \mathrm{GeV}$ CERN data. Thus it was clear that Feynman-Yang scaling was correct to first order; this scaling law was proposed independently by Feynman [14] and Yang [15] with totally different models. Yang's model was called Limiting Fragmentation and Feynman's model was just called Feynman's model; our ISR data showed that both models were correct.

In subsequent years, people worked very hard to find and then study small deviations from Feynman-Yang scaling; however, until 1971, there was absolutely no agreement about how inclusive cross-sections would behave in the $\mathrm{TeV}$ region. Thus, this first verification of Feynman-Yang scaling seems rather important; however, CERN never seemed to appreciate the importance of this result or of several other significant ISR results.

Now returning to Professor Sessler's lectures, he noted that Ken Robinson and Gus Voss, who were then at CEA, first developed the idea of low beta intersections. This technique involved installing quadrupoles near a collision point to squeeze the beams so that they are very small where they collide; this technique increases the luminosity without changing the intensity of the beams. These low beta intersections are now very important to all colliders.

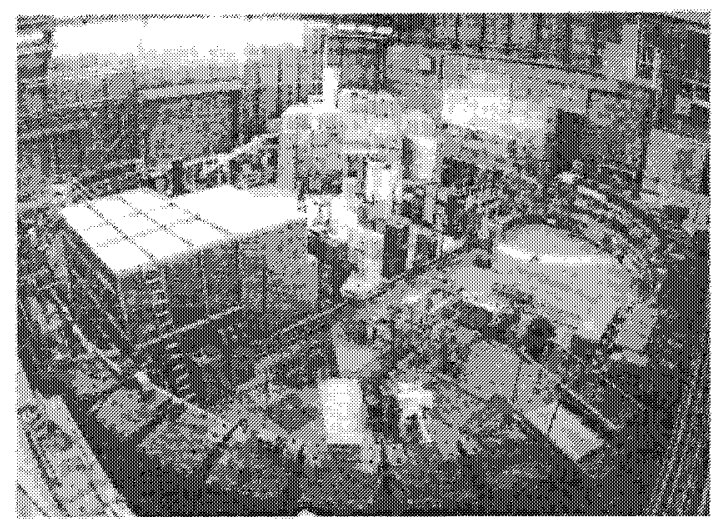

Fig. 26 ICE Storage Ring at CERN ${ }^{\dagger}$

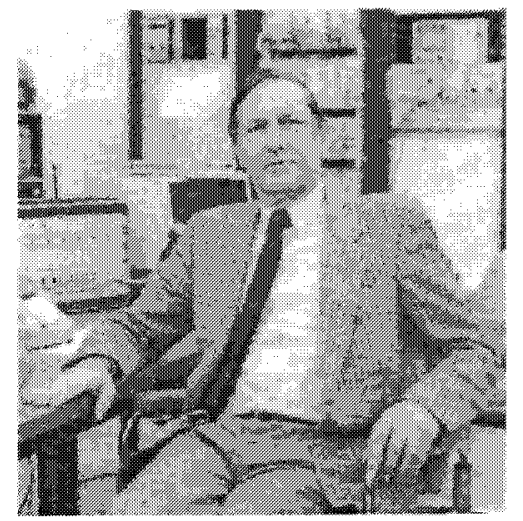

Fig. 27 Simon van der Meer ${ }^{\dagger}$

Another important idea that made colliders work was stochastic cooling. This was the second very clever idea of Simon van der Meer; he received the Nobel Prize for it. While the idea itself is rather simple, using it can be rather complex. I will not describe every detail of the technique, but basically one installs a detector on one side of a ring to determine if some part of the beam is properly centered or not; then, while the beam is going around to the ring's other side, one can send a signal across the ring's diameter. Because this signal can arrive before the beam arrives, the signal can tell the ring to energize some magnets, which then recenter the beam. Dr. van der Meer made a complex but very impressive device to test this technique at CERN, called the ICE storage ring, which is shown in 
Fig. 26. This stochastic cooling technique is especially important with antiprotons because they normally have a much lower intensity than protons; thus, being able to focus antiprotons to increase their brightness is very important. To improve the antiproton beam's longitudinal, transverse and vertical phase space, ICE had many detectors and many corrector magnets; each magnet was $180^{\circ}$ away from its detector. ICE worked beautifully. Stochastic cooling then allowed the SPS antiproton-proton collider to be built at CERN with enough luminosity to discover the intermediate boson. Simon van der Meer is shown in Fig. 27.

The Fermilab Tevatron-collider, which was the next big collider to operate, is shown in Fig. 28; it is just outside of Chicago. At the moment it is the world's highest energy collider; its c.m. energy is almost $900 \mathrm{GeV}$.

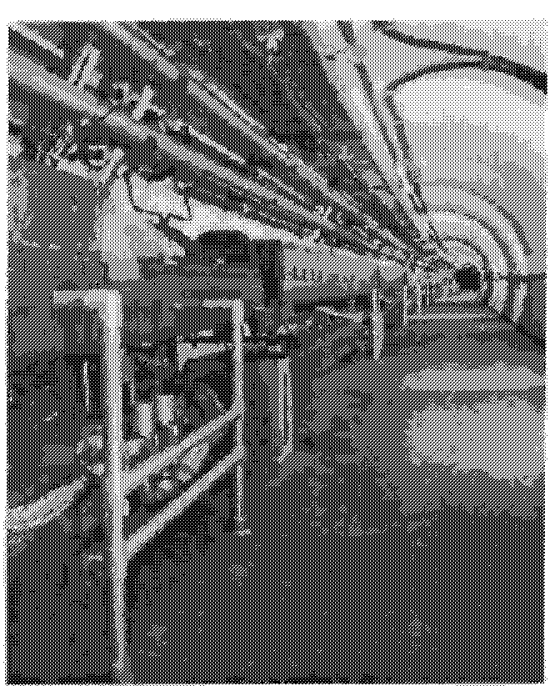

Fig. 28 Fermilab Tevatron Tunnel ${ }^{\dagger}$

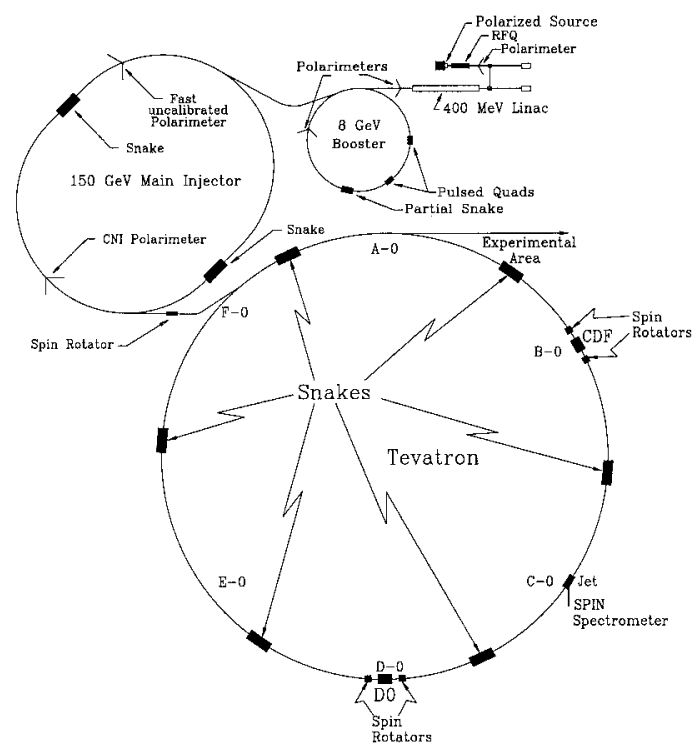

Fig. 29 Fermilab Design for Polarized Protons

Figure 29 is a drawing of Fermilab from our polarized Tevatron study [16]. Starting in 1991 Fermilab commissioned our SPIN Collaboration to design the capability to accelerate polarized protons in the Main Injector and the Tevatron; however, in 1995, Fermilab decided not to do it, at least for now. This was partly due to the $\$ 25$ Million cost, because there are no appropriate empty spaces for 6 Siberian snakes. Creating these 6 empty spaces would require removing 24 existing Tevatron superconducting ring dipoles and replacing them with shorter higher field dipoles. The Tevatron is about 6.3 kilometers in circumference with an $8 \mathrm{GeV}$ Booster and its new $150 \mathrm{GeV}$ Main Injector which started operating earlier this year. 
Another beautiful large collider is the HERA positron-proton collider at DESY which is shown in Fig. 30. HERA is similar in size to Fermilab because its proton energy is similar. HERA recently reached $920 \mathrm{GeV}$; thus its energy is now slightly higher than Fermilab's. No one has yet reached a TeV, but they are both getting close. HERA collides protons with either electrons or positrons. HERA's center of mass energy is much lower than Fermilab's because HERA's electrons or positrons are at about $30 \mathrm{GeV}$.

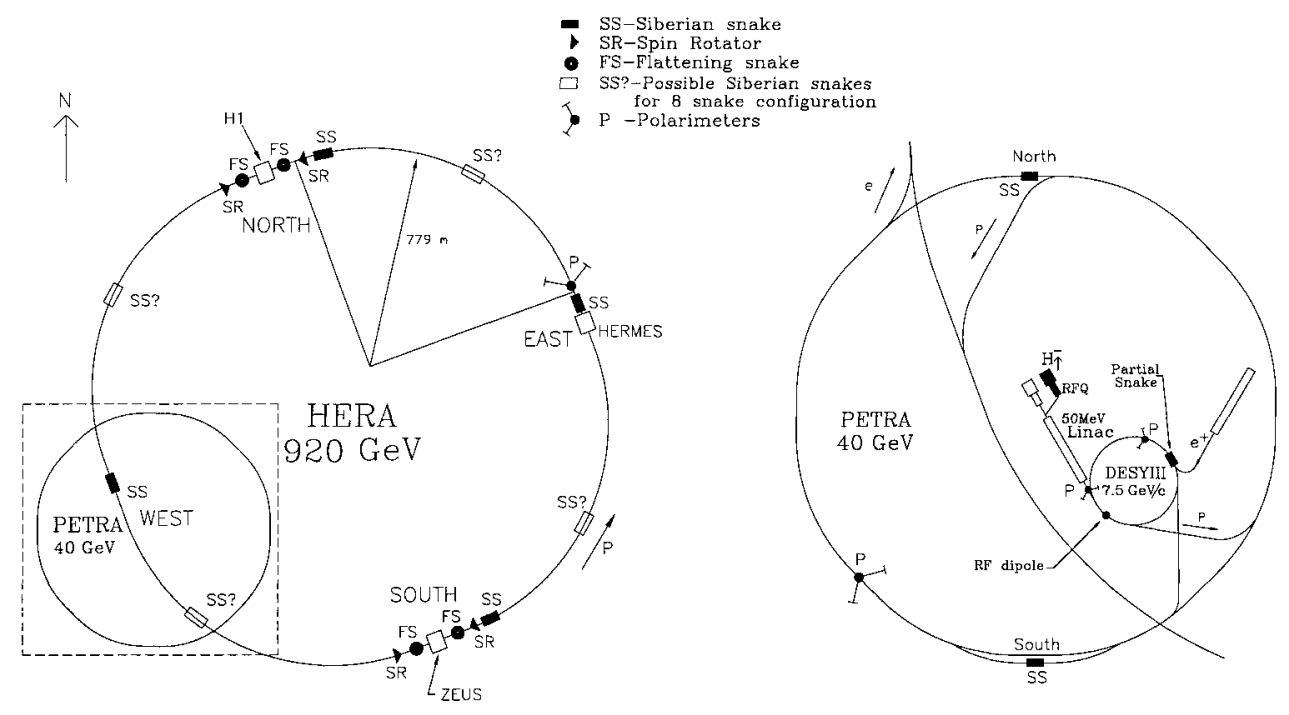

Fig. 30 HERA Design for Polarized Protons

Starting in 1996 we also did a polarized proton study for HERA, which already had polarized positrons. Professor Wilk and the other DESY Directors were interested in possibly polarizing the protons and they commissioned us to study the problem. We concluded that one could easily install four Siberian snakes in HERA; however, four snakes might not be enough to maintain the proton polarization at $920 \mathrm{GeV}[17]$. Eight Siberian snakes would certainly be able to maintain the polarization but would cost more money. Figure 30 shows the Siberian Snakes and other hardware needed to have polarized protons at $920 \mathrm{GeV}$; by looking at the names of all HERA's booster rings, one can see a history of DESY. The lowest energy booster is DESY III and then PETRA and then finally HERA itself; thus, we certainly recycle our old accelerators and do not waste them.

The second part of my talk is more directly related to this Conferences main subject, which is storage rings with internal targets. When Prof. Meyer asked me to give this talk, my first concern was what to talk about. Then I realized that I had worked on an early "storage ring" experiments for my thesis at the AGS in 1962-63. We used a rotating $\mathrm{CH}_{2}$ target about one millimeter thick placed inside the AGS ring to study proton-proton elastic scattering at high $P_{\perp}^{2}$. At that time, one did not think of the AGS as a storage ring, but it had a storage time of about 
one second, during which the beam passed through our target about 500,000 times and was effectively used up. The storage time was somewhat smaller than what is used now, but the AGS was indeed being used as a Storage Ring.

We published a Physical Review Letter [18] on the AGS experiment, which is shown in Fig. 31; since it was my thesis, I firmly believe that it was a nice experiment. Notice that it was submitted on 11 November 1963 and published on 1 December 1963. We later published a Physical Review paper in 1965 [19]. In those days, one really had to publish a detailed Physical Review paper after the PRL; otherwise the editor, Professor Goudsmit, would remember and the next time you submitted a Letter, he would return it without even reading it.

This AGS data is an important part of Fig. 32, which is a 1967 graph [20] of all high energy proton-proton elastic scattering cross-sections plotted against a scaled transverse momentum variable called $\beta^{2} P_{\perp}^{2}$ that I derived around 1964 [20] by assuming that protons behave as Lorentz-contracted spheres. This plot of all the world's high energy proton-proton data in 1967, was inspired by the sharp break found in our 1966 Argonne ZGS proton-proton elastic experiment at exactly $90_{\mathrm{cm}}^{\circ}$; that was probably the first evidence for structure inside the proton [21]. Figure 32 also contains the AGS data; note the point near $\beta^{2} P_{\perp}^{2}=13(\mathrm{GeV} / \mathrm{c})^{2}$; its $-\mathrm{t}$ value was about $25(\mathrm{GeV} / \mathrm{c})^{2}$. Since this point was measured 36 years ago, no one has measured an exclusive cross-section at a larger transverse momentum; perhaps, this is because our 1963 AGS experiment $[18,19]$ required a five-month dedicated run with an internal target.
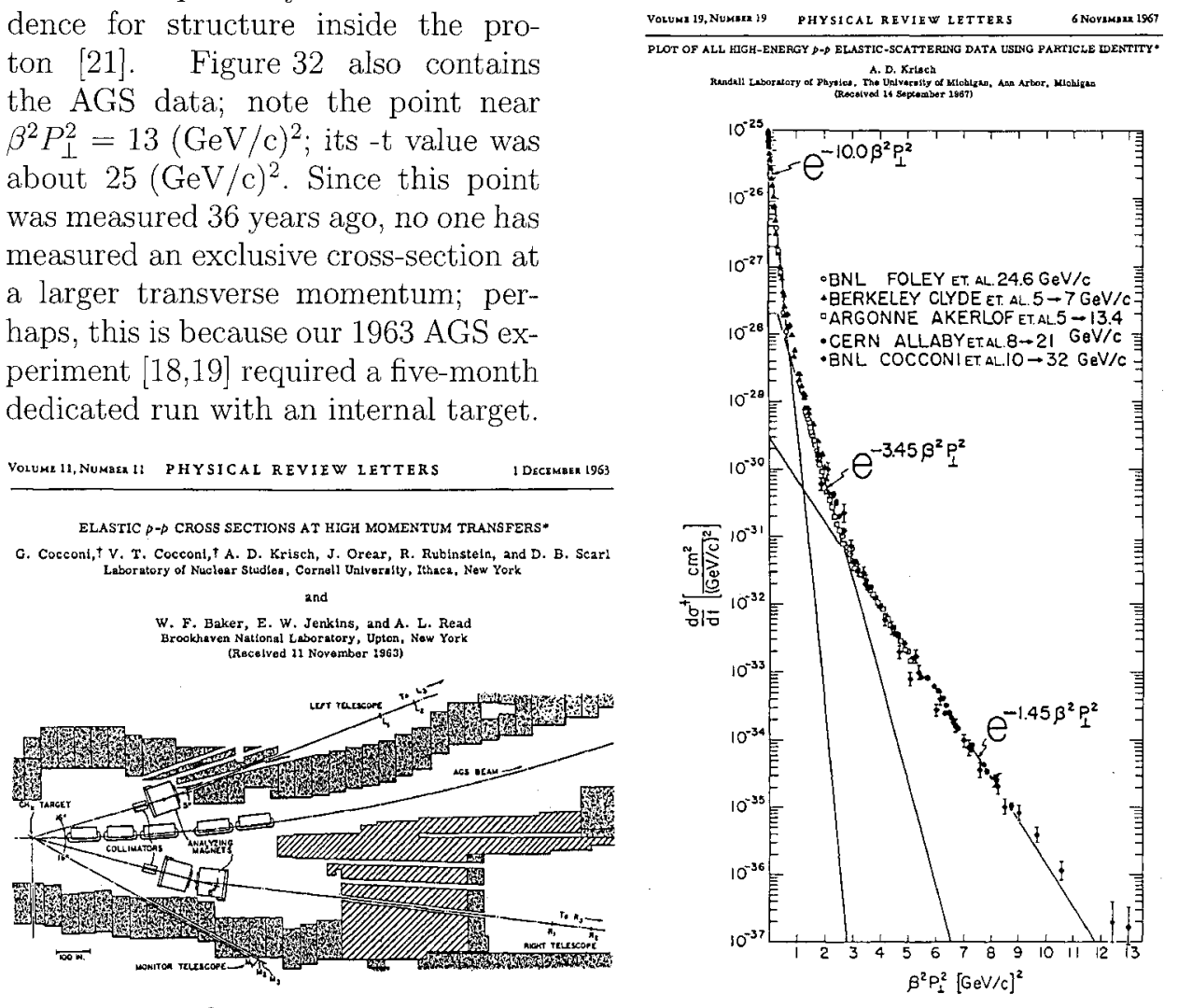

Fig. 31 High- $P_{\perp}^{2}$ Elastic p-p Experiment $[18,19]$

Fig. 321967 Plot of p-p Elastic Data [20] 
Now I will jump from 1963 to 1999 for the HERMES experiment; we heard two talks about HERMES this morning. During the last few years, HERMES has certainly been one of the most impressive fixed target storage ring experiments. The experimental apparatus is shown in Fig. 33; the HERMES group successfully designed and carried out this beautiful experiment with an impressive polarized "storage cell" target. The experiment has produced some important data on the nucleon structure functions. HERMES certainly has a very different scale in geometry and in time than the above experiments in the 1960's and early 1970's.

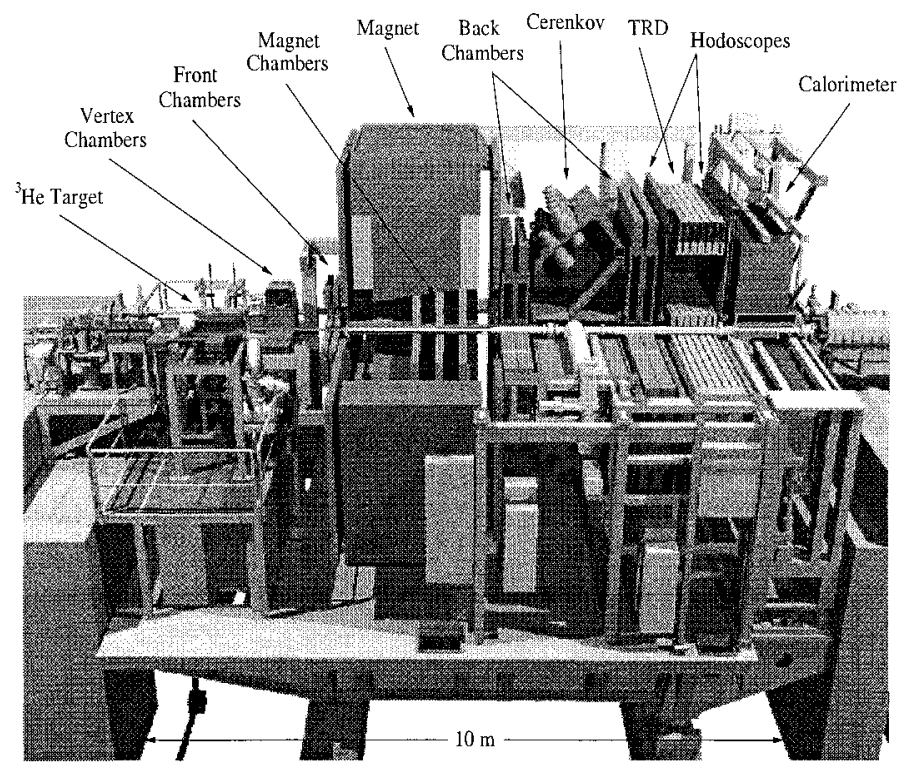

Fig. 33 The HERMES Experiment at HERA

The AmPS facility, which is shown in Fig. 34, has had internal targets and a Siberian Snake which allowed it to carry out some nice experiments. It is very sad that AmPS was recently shut down, like the ZGS, before it was ready to be shut down. I recall that in 1974, when the ZGS was already scheduled to be shut down, Professor Dirac visited Argonne and toured the ZGS; apparently, he was quite impressed and then said "You high energy physicists seem quite foolish; you build beautiful facilities and then shut them down before they have been half-utilized." Of course, Dirac was a theorist and theorists are sometimes not very practical, but perhaps he was wiser than some of our administrators and politicians.

The very nice COSY facility at Jülich is shown in Fig. 35. COSY operates both as a storage ring with some internal targets and as an extracted beam facility. Notice that it uses a cyclotron as its injector. COSY has been running for several years; it has outstanding hardware and it has the potential to become one of the worlds leading intermediate energy facilities. 


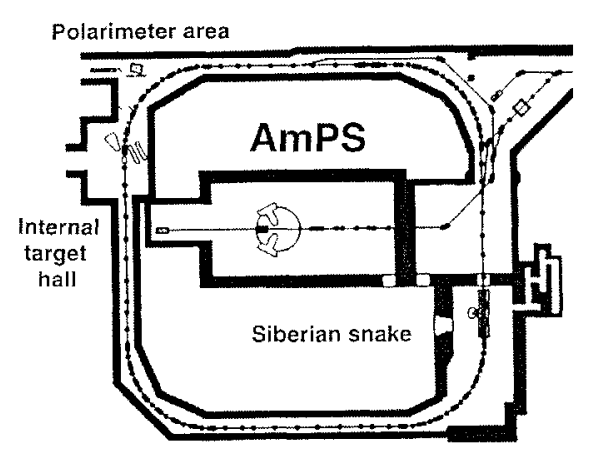

Fig. 34 AmPS at NIKHEF

Next, I will briefly discuss a few activities at the IUCF Cooler Ring. The first activity is the electron cooling work of Bob Pollock and his colleagues; in the 1980s, they used electron cooling to develop the pioneering IUCF Cooler Ring [22], which is shown in Fig. 36. Electron cooling was certainly invented by Budker and his colleagues at Novosibirsk; but the IUCF Cooler Ring demonstrated that electron cooling could provide a stored beam quality that has allowed experiments of unprecedented precision. It seems that the Indiana Cooler Ring has had a large influence over physics in many energy ranges.

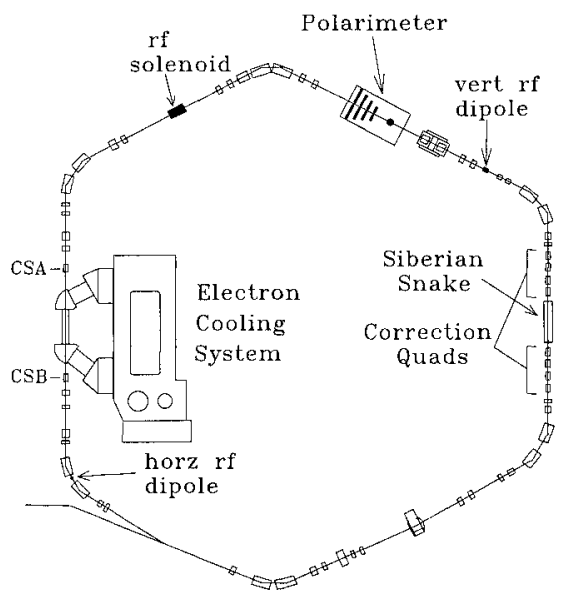

Fig. 36 IUCF Cooler Ring 1989. Figure 37 shows the data which first showed that a Siberian Snake really is capable of overcoming depolarized resonances [23]. Notice that with no snake, 
there is full polarization only if all imperfection fields are exactly corrected; any uncorrected imperfection field destroys the polarization. However, with the Siberian snake turned on, we completely overcame this $G \gamma=2$ imperfection depolarization.

Now I will move to the future. It would be difficult to cover all future storage ring facilities, but I will discuss some that I am familiar with. Starting at the very highest energy, I must mention the SSC. Many people would like to forget it; however, it was very important during 1983 to 1993 when we spent about $\$ 2$ Billion on it. As shown in Fig. 38, the SSC was huge; it was about 80 kilometers in circumference and contained many rings. The injector system is expanded to show its detail; there were three boosters: the $12 \mathrm{GeV}$ Low Energy Booster; the $200 \mathrm{GeV}$ Medium Energy Booster; and the $2 \mathrm{TeV}$ High Energy Booster. The two $20 \mathrm{TeV}$ Rings would allow many people to do experiments. We spin people feel

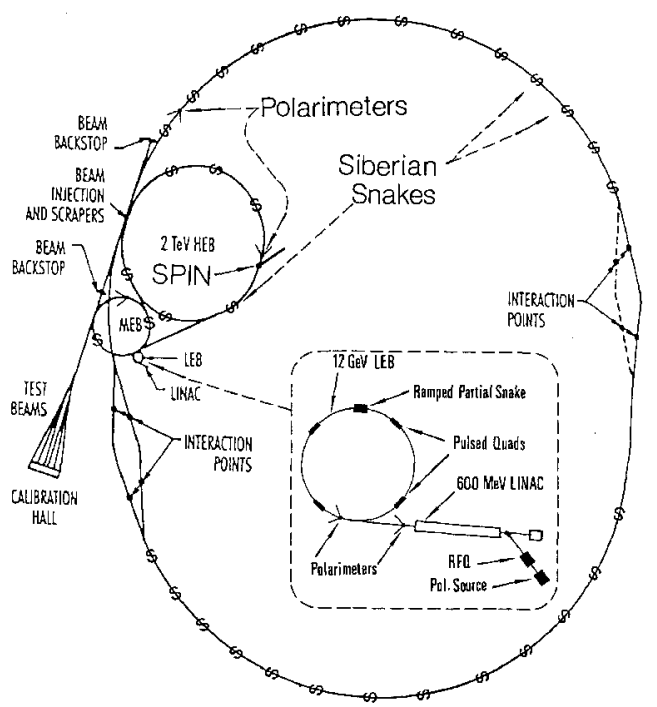

Fig. 38 SSC Design for Polarized Protons especially badly about the SSC's death, because it was modified to have twenty-six 19-meter-long empty spaces to later install 26 Siberian snakes in each SSC Ring. Of course, the SSC and its empty spaces for snakes are now all gone.

The highest energy facility now being constructed is the LHC at CERN which will be installed in the present LEP tunnel when LEP finishes running. CERN is now building the magnets for $\mathrm{LHC}$, which will be installed in the present $28 \mathrm{~km}$ LEP tunnel. Apparently, LHC should first operate for physics runs around 2006. LHC will allow the study of $7 \mathrm{TeV}$ on $7 \mathrm{TeV}$ proton-proton collisions with a luminosity of $10^{33}$ or $10^{34}$. LHC is certainly the largest presently approved collider; because it is quite expensive, many countries are contributing to its cost. Any larger facilities may be even more international because of their high cost.

The LEP electron-positron collider, which is now in this same $28 \mathrm{~km}$ circumference tunnel, has been running very effectively. It now operates at about $100 \mathrm{GeV}$ in each ring, which required overcoming a major synchrotron radiation problem. The main cost in CERN's electric bill is for the RF power to overcome LEPs synchrotron radiation; this requires almost 100 MegaWatts. LEP may have reached the upper limit for a circular electron ring, because the synchrotron radiation energy increases as the fourth power of the energy; therefore, doubling LEP's energy to $200 \mathrm{GeV}$, would increase its synchrotron radiation 16 -fold; this might require more than a TeraWatt of electric power, which does not seem practical. 
The UNK facility, at the Institute of High Energy in Physics in Protvino, Russia, was to be built in phases, which would eventually produce a $3 \mathrm{TeV}$ on $3 \mathrm{TeV}$ collider. The 21-kilometer-circumference UNK tunnel, which is shown in Fig. 39, is just slightly smaller than the LHC tunnel. However, IHEP was more conservative in its superconducting magnet design; UNK was designed to operate at about 4 Tesla, while CERN is now planning to run LHC at about 7.7 Tesla. UNK contains a huge underground area built for our NEPTUN/NEPTUN-A experiment; we would have used a polarized jet and a 55-meter-long spectrometer to study spin effects in high-P $P_{\perp}^{2}$ elastic proton-proton scattering, first at $400 \mathrm{GeV}$ and then at $3 \mathrm{TeV}$. Our Russian NEPTUN collaborators would have several other spectrometers pointing at the Michigan Ultra-cold Polarized Jet which recently started operating. The UNK tunnel is essentially finished and about $80 \%$ of the UNK-1 ring magnets are constructed and tested; however, around August 1998, UNK was put on hold by the Russian government due to the financial situation. About 100 people are maintaining the $21 \mathrm{~km}$ tunnel in the hope that IHEP may later finish and operate UNK, but the economic situation has already delayed UNK by 5 or 6 years.

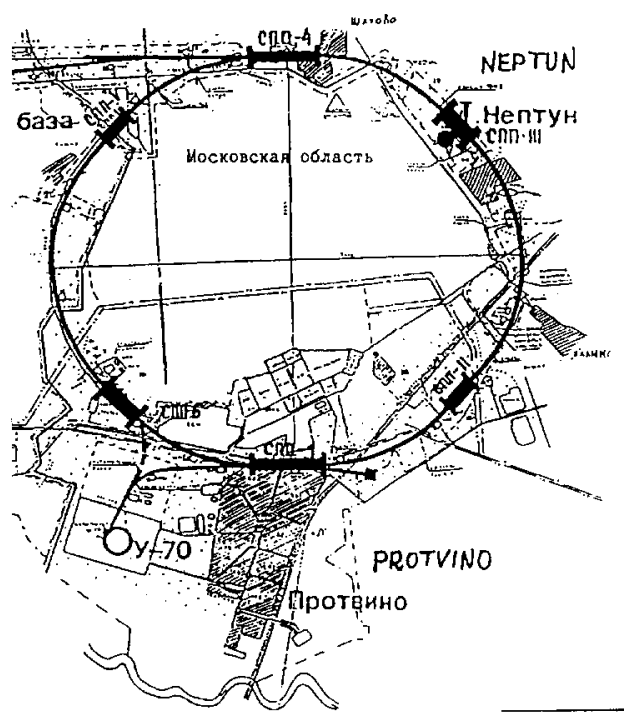

Fig. 39 UNK at IHEP-Protvino

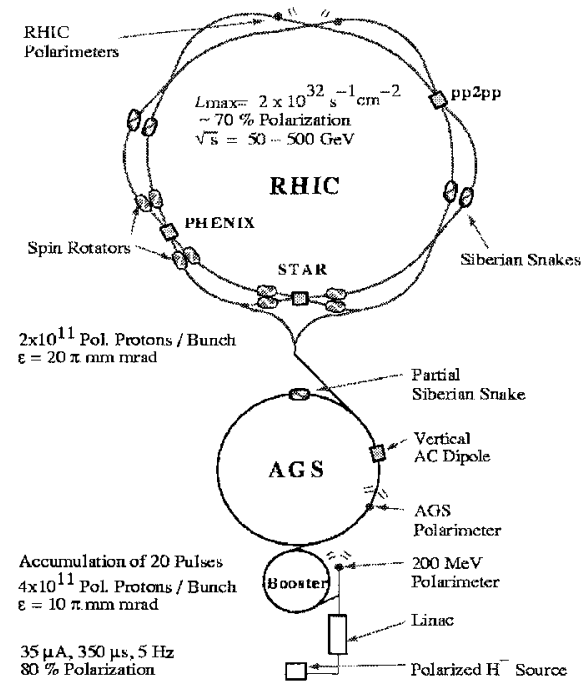

Fig. 40 RHIC with Polarized Protons

The RHIC facility is shown in Fig. 40. RHIC seems important for both nuclear and high energy physics, because it will accelerate and collide both Relativistic Heavy Ions and polarized protons. RHIC should have a rather high luminosity and it recently accelerated and stored heavy ions in one ring; the first heavy ion collisions should occur in December 1999. The first polarized proton commissioning run should occur in 2000 and the first polarized proton physics run should occur in 2001. During the next decade, RHIC should produce outstanding hadron scattering data in the $100 \mathrm{GeV}$ per nucleon range for both nuclear and high energy physicists. 
The next new storage ring is the MIT-Bates facility, which is shown in Fig. 41. I consider Bates as a future facility because the South Hall Storage Ring, which is a $1 \mathrm{GeV}$ polarized electron storage ring, should start operation early in 2000. In July 1999, the BLAST detector was being installed in the ring. This $1 \mathrm{GeV}$ polarized electron ring seems to have a great deal of capability.

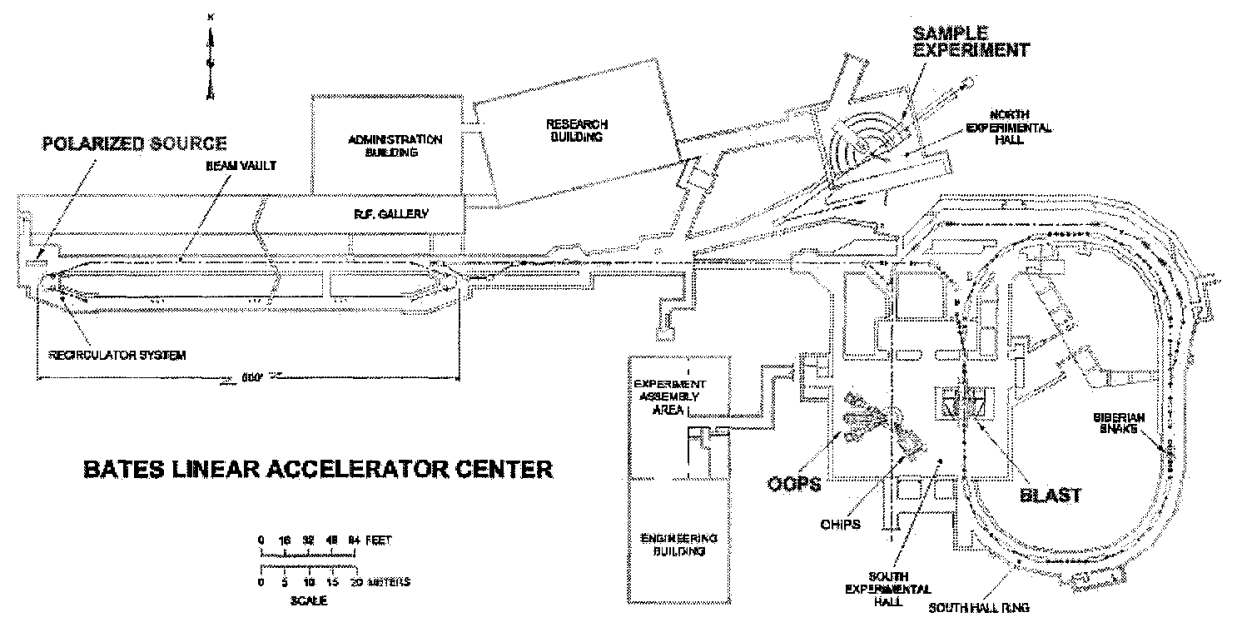

Fig. 41 MIT-Bates Linac and New Storage Ring

I will end with Fig. 42, which shows the Indiana University Cyclotron Facility Cooler Ring. The IUCF name has become a lie because the Cooler Ring no longer has a Cyclotron injector; the Cyclotron is now curing people with eye problems. The new injector for the Cooler Ring consists of two high quality new facilities.

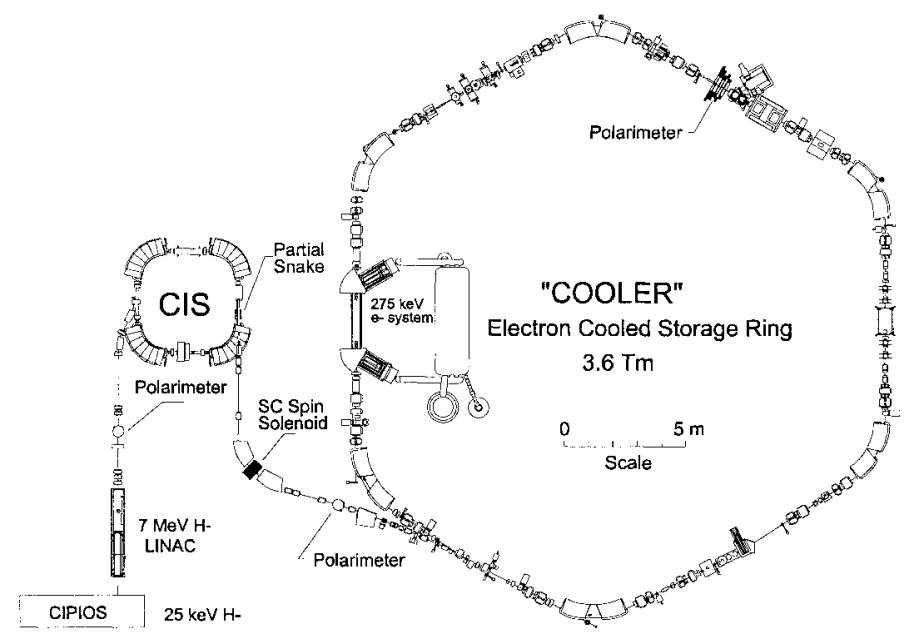

Fig. 42 IUCF's CIPIOS-CIS-Cooler Ring Complex 
One is the Cooler Injector Synchrotron (CIS), which is a new small Synchrotron that is a much better injector for the Cooler Ring than the Cyclotron. [A Cyclotron is not a good injector for a Synchrotron because of the bad energy match.] In July 1999 the new CIPIOS polarized ion source started operating; CIPIOS is coupled to CIS through a $7 \mathrm{MeV}$ RFQ and LINAC. The CIPIOS-CIS-Cooler Ring system is now working very well; there were several unpolarized runs in early 1999 and the first polarized run occurred in July 1999. The experimental hardware installed in the Cooler Ring includes: an Illinois laser-driven polarized target, a Wisconsin atomic-beam-source polarized target, our Siberian snake, Bob Pollock's plasma target, the neutron tagging experiment and Andy Bacher's future polarized deuteron experiment. Note that our conference chairman, Professor Meyer, is leading the three-body experiments with the Wisconsin target.

Both MIT-Bates and IUCF recently went through, what Richard Milner described to me as, a near-death experience when our government had decided to shut down Bates on rather short notice. However, by making an effective case for the importance of its physics capabilities, each laboratory managed to convince the government to substantially delay the shut-down date. Certainly, no laboratory, which requires a large budget, can expect to live forever. However, when we consider Bates and IUCF, together with HERA, COSY, RHIC, LHC and hopefully UNK and some other facilities, we can look forward to a broad range of exciting storage ring physics for many years.

\section{REFERENCES}

Note: In preparing this manuscript, I did not have time to find every detailed reference. For more detailed references, please consult Prof. Sessler's book [1] and the proceedings of this conference.

$\dagger$ Photo credits: From the private collection of A.M. Sessler.

1. A.M. Sessler, Lectures at Osaka, Washington and Vancouver; C.Pelegrini and A.M. Sessler, The Development of Colliders, AIP (1995).

2. A.W. Chao and M. Tigner, Handbook of Accelerator Physics and Engineering, page 12, World Scientific, Singapore (1999).

3. E.D. Courant, M.S. Livingston, and H.S. Snyder, Phys. Rev. 88, 1190 (1952).

4. D.W. Kerst et al., Fixed Field Alternating Gradient Particle Accelerators, 32, CERN Symposium (1956).

5. D.W. Kerst et al., Phys. Rev. 102, 590 (1956).

6. K. Johnsen, The CERN Intersection Storage Rings, 8th International Conference on High Energy Accelerators, CERN, 79 (1971).

7. G.K. O'Neill, Phys. Rev. 102, 1418 (1956).

8. D.B. Lichtenberg, R.G. Newton, and M.H. Ross, MURA Report-DBL/RGN/MHR-1 (April 26, 1956), unpublished.

9. C.W. Akerlof et al., Phys. Rev. D3, 645 (1971); L.G. Ratner et al., Phys. Rev. Lett. 16, 855 (1966), and Phys. Rev. 166, 1353 (1968). 
10. J.V. Allaby et al., CERN Report No. 70-12 (1970).

11. L.G. Ratner et al., Phys. Rev. Letters 27, 68 (1971).

12. L.G. Ratner et al., Phys. Rev. D9, 1135 (1974).

13. A. Bertin et al., Phys. Letters 38B, 260 (1972).

14. R.P. Feynman, Phys. Rev. Letters 23, 1415 (1969).

15. C.N. Yang et al., Phys. Rev. 188, 2159 (1969) and Phys. Rev. Lett. 25, 1072 (1970).

16. Acceleration of Polarized Protons to $120 \mathrm{GeV}$ and $1 \mathrm{TeV}$ at Fermilab, SPIN Collaboration, UM HE 95-09 (1995).

17. Acceleration of Polarized Protons to $920 \mathrm{GeV}$ at HERA, SPIN Collaboration, UM. HE 99-05 (1999).

18. G. Cocconi et al., Phys. Rev. Letters 11, 499 (1963).

19. G. Cocconi et al., Phys. Rev. 138, B165 (1965).

20. A.D. Krisch, Phys. Rev. Letters 19, 1149 (1967); Phys. Rev. 134, B1456 (1964), Phys. Rev, Letters 11, 217 (1963).

21. C.W. Akerlof, Phys. Rev. Letters 17, 1105 (1966); Phys. Rev. 159, 1138 (1967).

22. R.E. Pollock, IEEE Trans. Nucl. Sci. 30, 2056 (1983).

23. A.D. Krisch et al., Phys. Rev. Letters 63, 1137 (1989).

24. J.E. Goodwin et al., Phys. Rev. Letters 64, 2779 (1990). 

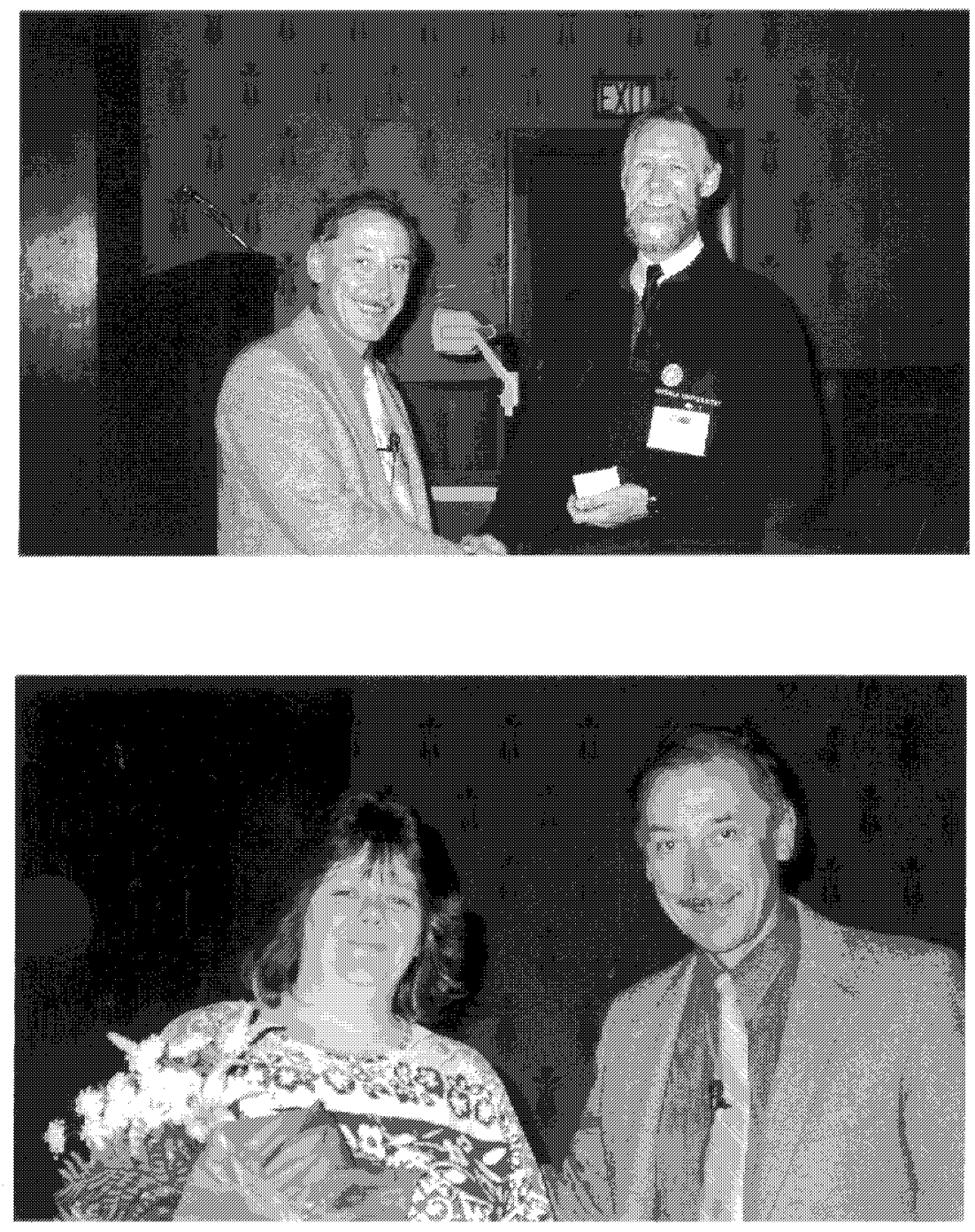